\title{
COVID-19 and acute pancreatitis: examining the causality
}

Enrique de-Madaria $\mathbb{1}^{1,2 凶}$ and Gabriele Capurso ${ }^{3}$

Case reports and retrospective cohort studies have reported an association between acute pancreatitis and COVID-19. As SARS-CoV-2 (the causative agent of COVID-19) receptors are expressed in the pancreas and endothelial damage can occur, this association is plausible. However, this hypothesis has many biases and needs further investigation.

Acute pancreatitis is a common disease, being the gastrointestinal disease most frequently requiring acute hospitalization $^{1}$. The coronavirus disease 2019 (COVID-19) pandemic (see COVID-19 dashboard) has now affected $>54$ million people and caused $>1.3$ million deaths worldwide in 2020. There are reports describing patients with COVID-19 and acute pancreatitis without a clear aetiology. This Comment aims to discuss whether COVID-19 should be considered as a new aetiology of acute pancreatitis. This question is not easy to answer: causality has troubled philosophers and health-care professionals for millennia ${ }^{2}$. In this case, the simplistic Koch's postulates are not useful, as we are addressing patients who have confirmed COVID-19, and acute pancreatitis might be an infrequent complication of the viral infection. As a systematic method to address this important question is needed, we have applied the Bradford Hill causality criteria framework to critically appraise the link between COVID-19 and acute pancreatitis ${ }^{2}$. These criteria make up the headings herein.

\section{Plausibility}

Severe acute respiratory syndrome coronavirus 2 (SARSCoV-2), the causative agent of COVID-19, uses ACE2 to enter human cells and TMPRSS2 for 'priming. Both of these proteins are highly expressed in gastrointestinal epithelial cells; infection of the intestinal epithelium has been confirmed in a brief communication ${ }^{3}$ and the virus can be easily isolated in stools. Pancreatic ductal, acinar and islet cells also express ACE2 (REF. ${ }^{4}$ ), so infection of the gland is plausible as the virus could spread from the duodenal epithelium to the pancreatic duct and then to acinar and islet cells. In a case report, SARS-CoV-2 has been isolated in samples from a pancreatic pseudocyst from a patient with acute pancreatitis ${ }^{5}$. Notably, SARS-CoV-2 is also able to cause diffuse severe endotheliitis of the submucosal vessels in several anatomical sites, and these changes, in turn, cause diffuse microischaemic disease ${ }^{6}$. Similar ischaemic damage could occur in the pancreas.

\section{Coherence}

Acute pancreatitis has been described as a complication of different viral infections in the past. SARS-CoV (the virus that causes severe acute respiratory syndrome, first detected in 2003) has been isolated in pancreatic tissue from autopsies ${ }^{7}$, but acute pancreatitis was not reported as a complication.

\section{Strength}

Strong associations are more likely to be causal than weak associations. Acute pancreatitis seems to be a very infrequent complication of COVID-19. If COVID-19 is associated with acute pancreatitis, an increase in otherwise idiopathic acute pancreatitis incidence is expected in patients with COVID-19. According to a retrospective cohort study analysing 11,883 hospitalized patients with COVID-19 from 12 hospitals in the USA, there were 32 cases of acute pancreatitis, yielding a point prevalence of $0.27 \%, 69 \%$ of them idiopathic ${ }^{8}$. A retrospective study involving more than 63,000 patients with COVID-19 from 50 Spanish emergency rooms before hospitalization reported a frequency of acute pancreatitis of $0.07 \%$; although the aetiology was not analysed in this study, COVID-19 was associated with a reduced risk of acute pancreatitis. An increase in the incidence of acute pancreatitis during the COVID-19 pandemic has not been firmly demonstrated.

\section{Consistency}

Cases of otherwise idiopathic acute pancreatitis in patients with COVID-19 were first published in China, and then throughout the world ${ }^{4,8}$. There have been case reports and retrospective cohort studies, but the populations under study, definitions of acute pancreatitis ${ }^{10}$ and results are heterogeneous, making it difficult to form firm conclusions.

\section{Specificity}

Acute pancreatitis is not specifically caused by SARS$\mathrm{CoV}-2$; it is a common disease with many causes, and the aetiology remains unknown in $15-25 \%$ of cases $^{1}$. 


\section{Box $1 \mid$ Open research questions}

- Is coronavirus disease 2019 (COVID-19) a new aetiology for acute pancreatitis? If so, how frequently does it complicate the course of COVID-19?

- In case of an association, can severe acute respiratory syndrome coronavirus 2 (SARS-CoV-2) infection cause acute pancreatitis without causing typical COVID-19 disease with respiratory symptoms, hence patients could present with an idiopathic acute pancreatitis and be recognized as SARS-CoV- 2 positive only by a screening swab?

- As acute pancreatitis has been reported during the course of COVID-19, are there other aetiologies - for example, drug-induced cases of acute pancreatitis in these patients - or is it a result of the SARS-CoV-2 infection itself?

- Is the putative mechanism for the association between acute pancreatitis and COVID-19 related to direct viral damage of pancreatic cells or to endothelial damage and/or thrombosis and ischaemic pancreatitis?

-What is the effect of acute pancreatitis on the prognosis of COVID-19?

Retrospective cohort studies ${ }^{8}$ and case-control studies have detected a higher proportion of idiopathic acute pancreatitis among patients with COVID-19. The preliminary results of an international multicentre retrospective and prospective case-control study, COVIDPAN (PSGBI webinar), which compared 149 patients with acute pancreatitis and COVID-19 and 1,628 patients with COVID-19-negative acute pancreatitis, showed a rate of idiopathic acute pancreatitis of $24 \%$ versus $14 \%$ respectively $(P=0.001)(M$. Nayar and S. Pandanaboyana; unpublished data).

Unfortunately, confounders and other biases cannot be ruled out in existing data. Some studies defined acute pancreatitis only by an increase in blood levels of pancreatic enzymes, but patients with COVID-19 can present with several complications that are associated with elevated blood levels of amylase and lipase, including acidosis, renal failure and diabetes ${ }^{10}$. Furthermore, patients with COVID-19 and acute pancreatitis, who might have pneumonia and are considered contagious, might not undergo tests such as endoscopic ultrasonography or MRI as frequently as patients with acute pancreatitis who are negative for COVID-19. The opportunity to diagnose microlithiasis (the most frequent final cause of initially idiopathic acute pancreatitis) or cystic lesions of the pancreas might, therefore, be missed, increasing the proportion of cases of unknown origin attributed to COVID-19 itself compared with patients without COVID-19 who undergo comprehensive aetiological diagnosis.

\section{Temporality}

The temporality of SARS-CoV-2 infection and the development of acute pancreatitis is heterogeneous. Some patients develop COVID-19 symptoms and abdominal pain at the beginning of the infection, and others have acute pancreatitis days after COVID-19 is diagnosed.

\section{Biological gradient}

It has not been consistently reported that acute pancreatitis is more frequent in patients with a higher SARS-CoV-2 viral load or severity of disease (which is linked to viral load). The COVIDPAN study suggests that acute pancreatitis in patients with COVID-19 is more frequently severe than in patients without
COVID-19, but this aspect is expected owing to the aggressive behaviour of COVID-19 itself, which is associated with multi-organ failure regardless of the presence of acute pancreatitis.

\section{Experimental evidence}

Animal models of COVID-19 developing acute pancreatitis are lacking. Owing to the rarity of acute pancreatitis as a complication of COVID-19, experimental evidence for a reduced incidence of acute pancreatitis due to prevention of COVID-19 is not possible.

\section{Conclusions}

Currently, the association between COVID-19 and acute pancreatitis is not based on evidence. The available data are difficult to interpret as, although acute pancreatitis is a relatively frequent disease, its occurrence in patients with COVID-19 seems rare and many questions remain (BOX 1). There is, therefore, a need for solid population-based epidemiological studies carefully addressing possible biases and taking into account the incidence of acute pancreatitis regardless of COVID-19 to show whether there is an increase in the incidence of acute pancreatitis compared with pre-COVID-19 times. Also, autopsy series of patients with COVID-19 with and without concomitant clinically evident acute pancreatitis could help in revealing the mechanisms behind this possible, but yet unproven, association. Finally, new animal models of COVID-19 are emerging that might help us to better understand the relationship between COVID-19 and pancreatitis.

1. Boxhoorn, L. et al. Acute pancreatitis. Lancet 396, 726-734 (2020).

2. Lucas, R. M. \& McMichael, A. J. Association or causation: evaluating links between "environment and disease". Bull World Health Organ. 83, 792-795 (2005)

3. Xiao, F. et al. Evidence for gastrointestinal infection of SARS-CoV-2. Gastroenterology 158, 1831-1833 (2020).

4. Liu, F. et al. ACE2 Expression in pancreas may cause pancreatic damage after SARS-CoV-2 infection. Clin. Gastroenterol. Hepatol. 18, 2128-2130 (2020).

5. Schepis, T. et al. SARS-CoV2 RNA detection in a pancreatic pseudocyst sample. Pancreatology 20, 1011-1012 (2020).

6. Pons, S., Fodil, S., Azoulay, E. \& Zafrani, L. The vascular endothelium: the cornerstone of organ dysfunction in severe SARS-CoV-2 infection. Crit Care 24, 353 (2020).

7. He, L. et al. Expression of the monoclonal antibody against nucleocapsid antigen of SARS-associated coronavirus in autopsy tissues from SARS patients [Chinese]. Di Yi Jun Yi Da Xue Xue Bao. 23, 1128-1130 (2020).

8. Inamdar S et al. Prevalence, risk factors, and outcomes of hospitalized patients with COVID-19 presenting as acute pancreatitis. Gastroenterology https://doi.org/10.1053/j.gastro.2020.08.044 (2020).

9. Miro, O. et al. Frequency of five unusual presentations in patients with COVID-19: results of the UMC-19-S1. Epidemiol. Infect. 148 , e189 (2020).

10. de-Madaria, E., Siau, K. \& Cardenas-Jaen, K. Increased amylase and lipase in patients with COVID-19 pneumonia: don't blame the pancreas just yet! Gastroenterology https://doi.org/10.1053/ j.gastro. 2020.04.044 (2020).

\section{Acknowledgements}

The authors thank M. Nayar and S. Pandanaboyana, from the Freeman Hospital (Newcastle upon Tyne, UK), for providing the preliminary results of the COVIDPAN study.

\section{Competing interests}

The authors declare no competing interests.

\section{RELATED LINKS}

COVID-19 dashboard: https://coronavirus.jhu.edu/map.html PSGBI webinar: https://www.psgbi.org/videos/recent-advances-in-acuteand-chronic-pancreatitis/ 